\title{
ANÁLISE MULTIVARIADA PARA ESTRATIFICAÇÃO VOLUMÉTRICA DE UMA FLORESTA OMBRÓFILA DENSA DE TERRA FIRME, AMAZÔNIA ORIENTAL ${ }^{1}$
}

\author{
Agostinho Lopes de Souza² e Deoclides Ricardo de Souza ${ }^{3}$
}

\begin{abstract}
RESUMO - O objetivo deste estudo foi propor um método de estratificação em classes homogêneas de estoque volumétrico da floresta ombrófila densa de terra firme não explorada, empregando-se as técnicas de análises de agrupamento e discriminante. A pesquisa foi conduzida na Unidade de Manejo Florestal (UMF) da Fazenda Tracajás $\left(02^{\circ} 35^{\prime} 53^{\prime}\right.$ 'S e 4747’10”W), pertencente à empresa Nova Era Agroflorestal, de propriedade do Grupo Rosa Madeireira, Município de Paragominas, Estado do Pará. Foi realizado um censo (100\%), no qual se estimaram os volumes do fuste comercial das árvores com dap $\geq 45 \mathrm{~cm}$ de 55 espécies comerciais em 49 talhões de exploração com 10 ha cada um. Reuniram-se os volumes estimados do fuste comercial das árvores individuais por talhão em ordem crescente. Elaborou-se uma matriz $X$ de dados desses volumes, em que cada variável $\mathrm{x}_{\mathrm{ij}}$ representou o i-ésimo volume classificado no j-ésimo talhão. A matriz $X$ foi utilizada como input nas análises de agrupamento e discriminante. A aplicação da análise de agrupamento, método de Ward, resultou em agrupamentos hierárquicos dos talhões em classes de estoques. A análise do dendrograma permitiu estratificar o povoamento em três grupos homogêneos e distintos, denominados classes I, II e III de estoques volumétricos. A análise discriminante, método de Fisher, indicou que $100 \%$ dos talhões foram corretamente classificados. A classificação multivariada da floresta em classes de estoques volumétricos mostrou-se um método eficiente na estratificação de áreas homogêneas de florestas ineqüiâneas, as quais podem se constituir em estratos, compartimentos, classes de sítio e unidades de produção anual.
\end{abstract}

Palavras-chave: Floresta tropical, estratificação, análise multivariada e classes de estoque.

\section{MULTIVARIATE ANALYSIS ON THE STRATIFICATION OF AN UNEXPLORED TERRA FIRME DENSE OMBROPHYLOUS FOREST, EASTERN AMAZON}

\begin{abstract}
ABSTRATCT - The objective of this study was to propose a method for stratification of an unexplored terra firme dense ombrophylous forest into classes of volumetric stocks, by using the techniques of cluster and discriminant analysis. The research was carried out at the Forest Management Unit (FMU) in Tracajás Farm (02 35'53" S and 47 47'10"W), owned by Nova Era Agroflorestal, in Paragominas, Pará, Brazil. An census (100\%) was accomplished in which the volumes of commercial whole-tree with dbh $\geq 45 \mathrm{~cm}$ were estimated for 55 commercial species in 49 stands 10 ha each. The estimated volumes of individual commercial wholetrees were organized in a increasing order. A X-matrix was built, in which each variable $x_{i j}$ represented the $i$-th volume classified in the $j$-th stand. This $X$-matrix was the input for the cluster and discriminant analysis. The application of the cluster analysis resulted in hierarchical clusters of the stands in stock classes. The analysis of the dendrogram allowed the stratification of the forest in three homogeneous and distinct groups, denominated classes of volumetric stock I, II and III. The discriminant analysis pointed out that 100\% of the stands were correctly classified. The multivariate classification of the forest into homogeneous classes of volumetric stocks showed to be an efficient method for stratification of uneven-aged forests, which can be constituted by extracts, compartments, site classes and annual production units.
\end{abstract}

Key words: Tropical forest, stratification, multivariate analysis and stock classes

\footnotetext{
${ }^{1}$ Recebido em 21.10.2003 e aceito para publicação em 10.11.2005.

${ }^{2}$ Departamento de Engenharia Florestal da Universidade Federal de Viçosa-UFV. E-mail: <alsouza@ mail.ufv.br>.

${ }^{2}$ Departamento de Engenharia Agronômica da Universidade Federal de Sergipe-UFS. E-mail:<souzadr@ @otmail.com>.
} 


\section{INTRODUÇÃO}

A floresta equatorial de terra firme apresenta elevada variabilidade, sobretudo de composição de espécies, grupos ecológicos, estruturas de idades, tamanhos e condições gerais de crescimento e produção (sítios), dentro de uma mesma comunidade florestal. Nesse caso, a classificação em áreas homogêneas permite inferir sobre a capacidade produtiva de cada zona ou classe de sítio e fundamentar a elaboração e execução dos planos de manejo sustentável.

Dentre os principais métodos usuais de classificação de sítio, podem-se citar: medições de fatores do sítio, como variáveis climáticas, edáficas e da própria vegetação; e medições de características da floresta natural, como o estoque de madeira produzida, as características de árvores e espécies de plantas ocorrentes na área (plantas indicadoras) (HUSCH et al., 1982).

As técnicas estatísticas multivariadas têm sido amplamente empregadas em estudos envolvendo simultaneamente variáveis de clima, solo, relevo, vegetação e geologia na classificação de áreas com cobertura florestal. Essas técnicas são utilizadas com objetivos básicos de ordenamento, visando determinar a influência de fatores do meio na composição e produtividade do local, e de agrupamento, com o propósito de classificação (BURTON et al., 1991).

Segundo Bowling e Zelazny (1992), a estatística multivariada permite a classificação de sítios em diferentes níveis de intensidade (regional, zonal e local). Para tanto, são definidas chaves individuais para a classificação dos tipos de vegetação, dos tipos e das propriedades dos solos, bem como de produtividade, criando-se uma rede para a classificação dos sítios.

A aplicação da estatística multivariada na área florestal é pouco freqüente, principalmente em estudos que envolvem a classificação multivariada de sítios (ASPIAZÚ, 1979; SOUZA, 1989; SOUZA et al., 1990; BRAGA, 1997; SOUZA et al., 1997; SOLOMÃO, 1998). Dentre as técnicas estatísticas multivariadas, as técnicas de análises de agrupamento e discriminante são as de maior viabilidade para a classificação de sítios homogêneos em florestas naturais inequiiâneas (SOUZA et al., 1990). O autor ainda afirmou que essas técnicas de classificação, denominadas análises de agrupamento e discriminante, relacionam-se com a separação de distintos indivíduos (árvores, parcelas e talhões) e com alocação desses em grupos predefinidos, de modo que a análise de agrupamento constrói os agrupamentos a partir das informações contidas na matriz de dados multivariados, e a análise discriminante aloca indivíduos em classes ou agrupamentos previamente estabelecidos.

No sentido de identificar diferentes sítios dentro de uma mesma comunidade vegetal, este estudo teve como objetivo propor um método de pós-estratificação da floresta ombrófila densa de terra firme não explorada, empregando-se as técnicas de análises de agrupamento e discriminante.

\section{MATERIAL E MÉTODOS}

A pesquisa foi realizada na unidade de manejo florestal (UMF) da Fazenda Tracajás (02³5' 53 ' S e 47\%47'10”W), pertencente à empresa Nova Era Agroflorestal, de propriedade do Grupo Rosa Madeireira, Município de Paragominas, Estado do Pará, Brasil. A tipologia florestal é classificada como floresta ombrófila densa de terra firme em estádio de sucessão primária (clímax).

Considerando que a cobertura florestal da unidade de manejo (UMF) apresenta grande variação em espécies, densidade, diâmetro, altura e volume para diferentes sítios dentro de uma mesma tipologia florestal, procurouse estratificar o povoamento em classes homogêneas de estoque volumétrico com potencial de exploração comercial.

Na execução do plano de manejo florestal, destinado à produção de madeira para serraria e laminação, foi realizado o censo (100\%), no qual se estimaram os volumes de fuste com casca das árvores individuais com dap $\geq 45 \mathrm{~cm}$ de 55 espécies comerciais autorizadas para corte pelo IBAMA, em 49 talhões de exploração de 10 hectares cada um, perfazendo um total de 490 hectares.

No censo (inventário $100 \%$ ) das árvores comerciais com dap $\geq 45 \mathrm{~cm}$ (diâmetro mínimo de corte), foram identificadas e marcadas as árvores-matriz, as árvores localizadas nas áreas de proteção ambiental e aquelas selecionadas para corte. Reuniram-se os volumes estimados de fuste comercial dessas árvores por talhão, em ordem crescente. Elaborou-se uma matriz $X$ de dados desses volumes, em que cada variável $\mathrm{x}_{\mathrm{ij}}$ representou o i-ésimo volume classificado no j-ésimo talhão.

A matriz $X$ foi utilizada como input nas análises de agrupamento e discriminante. Utilizaram-se a distância euclidiana simples e o método de Ward, com o emprego das expressões: 


$$
\begin{aligned}
d_{i j} & =\sqrt{\sum_{h=1}^{p}\left(x_{h i}-x_{h j}\right)^{2}}, h=1,2, \cdots, p \\
d_{i j}^{2} & =\left(\bar{x}_{i}-\bar{x}_{j}\right)^{2}
\end{aligned}
$$

em que $d_{i j}=$ distância euclidiana estimada entre os talhões i e $\mathrm{j}, \mathrm{x}_{\mathrm{hi}}=$ volume no talhão $\mathrm{i}, \mathrm{x}_{\mathrm{hj}}=$ volume no talhão j e d ${ }^{2}$ ij $=$ distância entre as médias das classes de estoque I e $\mathbf{J}$.

Para a definição das classes homogêneas de estoque volumétrico, traçou-se a linha de corte ou linha "fenon", que é uma linha paralela ao eixo horizontal do dendrograma obtido da análise de agrupamento (SOUZA et al., 1990).

A análise discriminante foi utilizada para verificar a distinção e classificação das classes homogêneas de estoque volumétrico obtidas pela análise de agrupamento.

$\mathrm{Na}$ análise de agrupamentos, os talhões ou unidades de trabalho formaram grupos homogêneos e distintos, agrupados nas classes I, II e III de estoques volumétricos.

Em cada classe de estoque foram instaladas aleatoriamente cinco parcelas de 100 x $100 \mathrm{~m}$ ( 1 ha) cada uma, para medição dos indivíduos com dap $\geq$ $15 \mathrm{~cm}$. No centro de cada parcela foi instalada uma subparcela de 10 x 100 m (0,1 ha), para a medição dos indivíduos com $5 \mathrm{~cm} \leq$ dap $<15 \mathrm{~cm}$.

Nas parcelas foram feitas as seguintes avaliações para cada árvore (SOUZA, 2003): nomes científico e vulgar, medição do diâmetro a 1,30 m do solo (dap), alturas total (ht) e comercial (hc), qualidade de fuste (QF), iluminação de copa (IC), cobertura de copa (CC), infestação de cipós (C) e danos naturais (D).

As espécies foram identificadas no campo, porém os indivíduos não identificados no local tiveram seus materiais botânicos coletados para identificação no Herbário do Museu Paraense Emílio Goeldi (MPEG), Estado do Pará.

No cálculo do número de parcelas, considerouse um erro de amostragem máximo de 20 a $95 \%$ de probabilidade (IBAMA, 1998).

A composição florística foi analisada com base na distribuição dos indivíduos em espécies (S) e por meio dos índices de diversidade máxima $\left(\mathrm{H}_{\text {máx }}\right)$ e de Shannon-Weaver (H'), conforme Brower e Zar (1984).
O volume de fuste com casca de árvores individuais foi estimado pelo emprego da equação $\hat{Y}=0,0774759688+0,517896768\left(\right.$ dap $\left.^{2} \times H c\right) \quad$ com $\mathrm{R}^{2}=0,9652$ e F=2994,78, desenvolvida por Queiroz (1984).

\section{RESULTADOS E DISCUSSÃO}

O dendrograma obtido da análise de agrupamento (Figura 1) apresenta no eixo vertical a distância euclidiana simples, em porcentagem, variando de zero a 100, e no eixo horizontal os talhões com seus volumes, formando as classes homogêneas de estoque volumétrico.

$\mathrm{Na}$ análise do dendrograma (Figura 1) foi traçada uma linha de corte no nível de homogeneidade de $20 \%$, destacando-se três grupos distintos, denominados classes I, II e III de estoques volumétricos, sendo a classe I de estoque formada pelos talhões I10 a I8, a classe II de estoque pelos talhões J3 a I13 e a classe III de estoque pelos talhões L14 a I5.

As classes I, II e III de estoques volumétricos englobaram talhões com baixo, médio e alto estoques volumétricos, com os respectivos volumes mínimo, médio, máximo e os desvios-padrão (Quadro 1).

A análise discriminante apresentou probabilidades de 0,30;0,37; e 0,33, respectivamente, nas classes I, II e III de estoques volumétricos. O procedimento final de classificação indicou que $100 \%$ dos talhões foram corretamente classificados nas classes de estoque (Quadro 2).

A classificação multivariada da floresta em classes I, II e III de estoques volumétricos mostrou-se um método eficiente na estratificação de áreas homogêneas de florestas ineqüiâneas, que podem se constituir em estratos, compartimentos, classes de sítio e unidades de produção anual (UPA). Esse método pode ter aplicação em inventário florestal, na elaboração e execução de planos de manejo, na delimitação de zonas de florestas de produção e proteção e, sobretudo, em estudos fitossociológicos e ambientais, em geral.

A aplicação da classificação multivariada em inventário florestal pode ser constatada pelo método de amostragem aleatória estratificada, no qual se estimaram os volumes do fuste comercial das árvores com dap $\geq 15 \mathrm{~cm}$ (Quadro 3). Foram inventariadas 15 parcelas de 100 x $100 \mathrm{~m}$ (1,0 ha), distribuídas aleatoriamente na área total de 490 hectares.

R. Árvore, Viçosa-MG, v.30, n.1, p.49-54, 2006

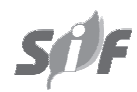




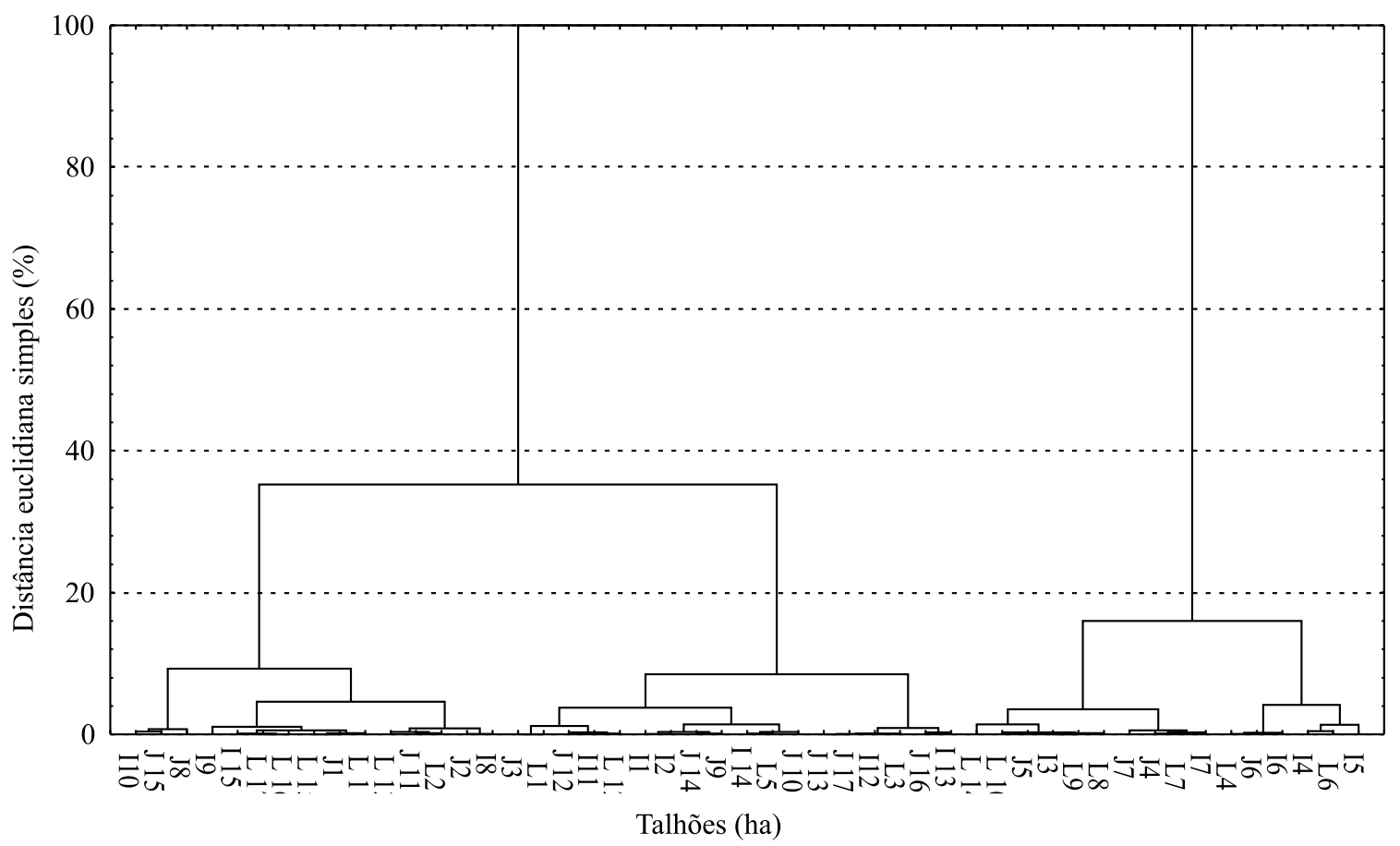

Figura 1 - Dendrograma mostrando os grupos de talhões representando as classes de estoques volumétricos, obtidos da análise de agrupamento utilizando a distância euclidiana simples e o método de Ward, Fazenda Tracajás, Município de Paragominas, Estado do Pará.

Figura 1 -Dendrogram of stand clusters showing the volumetric stock classes, obtained from cluster analysis using Euclidean distance and Ward method, Tracajás Farm, municipality of Paragominas, Pará State.

Tabela 1 - Caracterização das classes de estoque volumétrico, mediante os valores dos volumes mínimo, médio e máximo e os desvios-padrão

Table 1 -Characterization of volumetric stock classes, showing minimum, medium and maximum volumes and standard deviations

\begin{tabular}{ccccc}
\hline Classe de & \multicolumn{4}{c}{ Volume $\left(\mathrm{m}^{3} / \mathrm{ha}\right)$} \\
\cline { 2 - 5 } Estoque & Mínimo & Médio & Máximo & Desvio-Padrão \\
\hline I & 35,60 & 45,16 & 50,89 & 4,67 \\
II & 52,55 & 58,11 & 63,82 & 3,41 \\
III & 69,50 & 76,77 & 89,36 & 6,37 \\
\hline
\end{tabular}

A estratificação de uma população florestal em subpopulações homogêneas (Quadro 3) resultou na redução da variância total e das variâncias dentro de cada classe homogênea de estoque, com o aumento da precisão das estimativas e conseqüente redução no tamanho da amostra.

A média estratificada da população foi de 330,98 $\mathrm{m}^{3}$ e o erro-padrão da média, $18,38 \mathrm{~m}^{3} / \mathrm{ha}$. O erro de amostragem encontrado foi de 12 a $95 \%$ de probabilidade e atendeu aos critérios estabelecidos para inventariar florestas tropicais (erro inferior a 20\%).

Quadro 2 - Número de classes de estoque e porcentagens de classificação correta obtidas da análise discriminante Table 2 - Number of stock classes and percentages of correct classification obtained through the discriminant analysis

\begin{tabular}{cccccc}
\hline Classe de Estoque & \multicolumn{2}{c}{ Classificação em Classes de Estoque } & & Total & Classificação (\%) \\
\cline { 2 - 4 } & $\mathrm{I} P=0,30$ & II $\mathrm{P}=0,37$ & III $\mathrm{P}=0,33$ & & \\
\hline I & 15 & 0 & 0 & 15 & 100 \\
II & 0 & 18 & 0 & 18 & 100 \\
III & 0 & 0 & 16 & 16 & 100 \\
\hline Total & 15 & 18 & 16 & 49 & 100 \\
\hline
\end{tabular}


Quadro 3 - Distribuição dos volumes ( $\mathrm{m}^{3} / \mathrm{ha}$ ) das árvores com dap $\geq 15 \mathrm{~cm}$ por parcela e por classes de estoques volumétricos Table 3 - Distribution of volume $\left(\mathrm{m}^{3} / \mathrm{ha}\right)$ of trees with $\mathrm{dbh} \geq 15 \mathrm{~cm}$ per sample plot and per volumetric stock classes

\begin{tabular}{|c|c|c|c|c|}
\hline \multirow{3}{*}{$\begin{array}{l}\text { Parcela } \\
\text { (há) }\end{array}$} & \multicolumn{3}{|c|}{ Classes de Estoques Volumétricos } & \multirow[t]{3}{*}{ Total } \\
\hline & Classe I & Classe II & Classe III & \\
\hline & $\mathrm{m}^{3} / \mathrm{ha}$ & $\mathrm{m}^{3} / \mathrm{ha}$ & $\mathrm{m}^{3} / \mathrm{ha}$ & \\
\hline 1 & 396,657 & 379,125 & 261,089 & \\
\hline 2 & 325,496 & 281,278 & 360,213 & \\
\hline 3 & 271,891 & 219,074 & 348,115 & \\
\hline 4 & 367,719 & 460,282 & 231,611 & \\
\hline 5 & 431,675 & 301,888 & 342,404 & \\
\hline $\mathrm{A}_{\mathrm{j}}$ & $\overline{150}, \overline{00}$ & $\overline{180}, \overline{00}$ & $\overline{16} \overline{0,0} \overline{0}$ & $\overline{490,00}$ \\
\hline $\mathrm{N}_{j}^{\mathrm{j}}$ & 150,00 & 180,00 & 160,00 & 490,00 \\
\hline $\mathrm{n}_{\mathrm{j}}^{\mathrm{j}}$ & 5,00 & 5,00 & 5,00 & 15,00 \\
\hline$P_{j}^{j}$ & 0,30 & 0,37 & 0,33 & 1,00 \\
\hline $\bar{y}_{j}$ & 358,69 & 328,33 & 308,69 & \\
\hline$S_{j}$ & 62,22 & 93,33 & 58,21 & \\
\hline
\end{tabular}

$\mathrm{A}$ = área total da j-ésima classe de estoque, $\mathrm{N}_{\mathrm{j}}=$ número total de parcelas na $\mathrm{j}$-ésima classe de estoque, $\mathrm{n}_{\mathrm{j}}=$ número de parcelas medidas na j-ésima classe de estoque, $\mathrm{P}_{\mathrm{j}}=$ proporção na $\mathrm{j}$-ésima classe de estoque, $\bar{y}_{j}=$ média da amostra na $\mathrm{j}$-ésima classe de estoque e $\mathrm{S}_{\mathrm{j}}=$ desviopadrão da j-ésima classe de estoque.

No nível I de inclusão ( dap $\geq 15 \mathrm{~cm}$ ), os maiores valores de riqueza em espécies (S), densidade absoluta ( $\mathrm{n} / \mathrm{ha})$, volume de fuste comercial $\left(\mathrm{m}^{3} / \mathrm{ha}\right)$, diversidade máxima $\left(\mathrm{H}_{\text {máx }}\right)$ e Shannon-Weaver $\left(\mathrm{H}^{\prime}\right)$ foram para a classe I de estoque (Quadro 4). Os valores de diversidade máxima $\left(\mathrm{H}_{\text {máx }}\right)$ foram praticamente iguais nas classes I, II e III, apostando que essas áreas homogêneas de florestas diferem muito pouco em riqueza de espécies.

No nível II de inclusão ( $5 \mathrm{~cm} \leq$ dap $<15 \mathrm{~cm})$, a classe III de estoque foi a que apresentou maiores valores de riqueza em espécies (S), densidade absoluta (n/ha), dominância absoluta ( $\left.\mathrm{m}^{2} / \mathrm{ha}\right)$, volume de fuste comercial $\left(\mathrm{m}^{3} / \mathrm{ha}\right)$, diversidade máxima $\left(\mathrm{H}_{\text {máx }}\right)$ e Shannon-Weaver (H') (Quadro 5). Os valores de diversidade máxima $\left(\mathrm{H}_{\text {máx }}\right)$ foram iguais nas classes I e III de estoque, mostrando que essas áreas homogêneas de florestas não diferem em riqueza de espécies.

\section{CONCLUSÕES}

- A classificação multivariada dos talhões em classes de estoques volumétricos é um método eficiente na estratificação de florestas tropicais naturais.

- A estratificação dos talhões com baixo, médio e alto estoques volumétricos permite melhor planejamento e controle da produção florestal, bem como a execução das atividades de colheita, de tratamentos silviculturais e de monitoramento ou inventário florestal contínuo.

- O método de estratificação proposto permite também maior precisão das estimativas do inventário florestal e conseqüente redução de tempo e de recursos humanos e financeiros na execução dos levantamentos feitos por amostragem em florestas tropicais naturais.

\section{REFERÊNCIAS BIBLIOGRÁFICAS}

ASPIAZÚ, C. Classificação de sítios florestais mediante três métodos de análise da vegetação natural. Revista Árvore, v.3, n.1, p. 1-15, 1979.

BOWLING, C.; ZELANZNY, V. Forest site classification in New Brunswick. The Forestry Chronicle, v.68, n.1, p. 34-41, 1992.

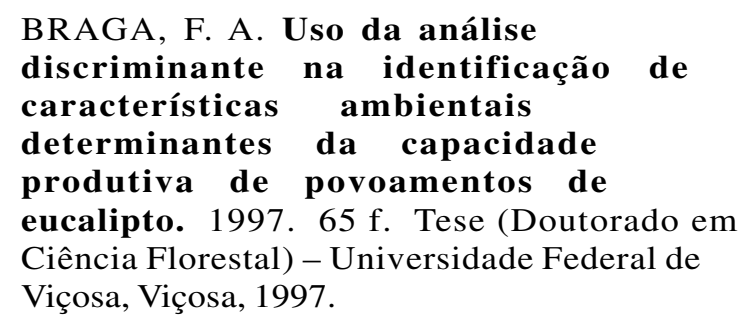

BROWER, J. E.; ZAR, J. H. Field \& laboratory methods for general ecology. 2.ed. Iowa: Northern Illinois University, 1984. 226p.

BURTON, A. J.; RAMM, C. W.; PREGITZER, K. S. Use of multivariate methods in forest research site selection. Canadian Journal Forestry

Research, v.21, p.1573-1580, 1991. 
HUSCH, B.; MILLER, C. I.; BEERS, T. W. Forest mensuration. 3.ed. New York: John Wiley \& Sons, 1982. $402 \mathrm{p}$.

IBAMA. Instrução Normativa № 6, de 28 de dezembro de 1998. 2p.Brasília: 1998.

MUELLER-DOMBOIS, D.; ELLEMBERG, H. Aims and methods of vegetation ecology. New York: Jonh Wiley \& Sons, 1974. 547 p.

QUEIROZ, W. T. Análise de fatores ("Factor Analysis") pelo método da máxima verossimilhança: aplicação ao estudo da estrutura de florestas tropicais. 1984. $109 \mathrm{f}$. Tese (Doutorado em Engenharia Florestal) - Universidade Federal do Paraná, Curitiba, 1984.

SOlOMÃo, A. L. Subsídios técnicos para elaboração do plano de manejo da floresta nacional do Rio Preto-ES. 1998. 151 f. Tese (Doutorado em Ciência Florestal) Universidade Federal de Viçosa, Viçosa, 1998.
SOUZA, A. L. Análise multivariada para manejo de florestas naturais: alternativas de produção sustentada de madeiras para serraria. 1989. 255 f. Tese (Doutorado em Ciência Florestal) - Universidade Federal do Paraná, Curitiba, 1989.

SOUZA, A. L. et al. Análises multivariadas para manejo de floresta natural na Reserva Florestal de Linhares, Espírito Santo: análises de agrupamento e discriminante. Revista Árvore, v.14, n.2, p.85-101, 1990.

SOUZA, A. L.; FERREIRA., R. L. C.; XAVIER, A. Análise de agrupamento aplicada à área florestal. Viçosa: SIF, 1997. 109 p. (Boletim Técnico SIF, 16).

SOUZA, D. R. Sustentabilidade ambiental e econômica do manejo em floresta ombrófila densa de terra firme, Amazônia Oriental. 2003. $123 \mathrm{f}$. Tese (Doutorado em Ciência Florestal) Universidade Federal de Viçosa, Viçosa, 2003. 


\section{ANEXOS}

Quadro 4 - Estimativas do número de espécies (S), densidade absoluta (n/ha), dominância absoluta (m²/ha), volume de fuste $\left(\mathrm{m}^{3} / \mathrm{ha}\right)$, diversidade máxima $\left(\mathrm{H}_{\text {máx }}\right)$ e o índice de Shannon-Weaver $\left(\mathrm{H}^{\prime}\right)$, por classe de estoque volumétrico, nível I de inclusão, Fazenda Tracajás, município de Paragominas, Estado do Pará

Table 4 - Estimatives of species number $(S)$, absolute density $(\mathrm{n} / \mathrm{ha})$, absolute dominance $\left(\mathrm{m}^{2} / \mathrm{ha}\right)$, whole tree volume, maximal diversity $\left(H_{\max }\right)$ and diversity index of Shannon-Weaver $\left(H^{\prime}\right)$, per volumetric stock class, level I inclusion, Tracajás Farm, municipality Paragominas, State Pará

\begin{tabular}{ccccccc}
\hline Classe de Estoque & $\mathrm{S}$ & $\mathrm{n} / \mathrm{ha}$ & $\mathrm{m}^{2} / \mathrm{ha}$ & $\mathrm{m}^{3} / \mathrm{ha}$ & $\mathrm{H}_{\text {max }}$ & $\mathrm{H}^{\prime}$ \\
\hline I & 163 & 322,4 & 27,36 & 358,69 & 5,10 & 3,98 \\
II & 153 & 309,0 & 27,45 & 328,33 & 5,00 & 3,80 \\
III & 141 & 313,8 & 25,88 & 308,69 & 5,00 & 3,83 \\
\hline Total & 218 & 315,0 & 26,90 & 331,90 & 5,39 & 3,97 \\
\hline
\end{tabular}

Quadro 5 - Estimativas do número de espécies (S), densidade absoluta (n/ha), dominância absoluta ( $\mathrm{m}^{2} / \mathrm{ha}$ ), volume de fuste $\left(\mathrm{m}^{3} / \mathrm{ha}\right)$, diversidade máxima $\left(\mathrm{H}_{\text {máx }}\right)$ e o índice de Shannon-Weaver $\left(\mathrm{H}^{\prime}\right)$, por classe de estoque volumétrico, nível II de inclusão, Fazenda Tracajás, município de Paragominas, Estado do Pará

Table 5 - Estimatives of species number, absolute density $(\mathrm{n} / \mathrm{ha})$, absolute dominance $\left(\mathrm{m}^{2} / \mathrm{ha}\right)$, stems volume, maximal diversity $\left(H_{\max }\right)$ and diversity index of Shannon-Weaver $\left(H^{\prime}\right)$, per volumetric stock class, level II inclusion; Tracajás Farm, municipality of Paragominas, State Pará

\begin{tabular}{ccccccc}
\hline Classe de Estoque & $\mathrm{S}$ & $\mathrm{n} / \mathrm{ha}$ & $\mathrm{m}^{2} / \mathrm{ha}$ & $\mathrm{m}^{3} / \mathrm{ha}$ & $\mathrm{H}_{\max }$ & $\mathrm{H}^{\prime}$ \\
\hline I & 112 & 846 & 4,80 & 93,98 & 4,72 & 3,93 \\
II & 103 & 854 & 4,93 & 91,23 & 4,63 & 3,85 \\
III & 113 & 886 & 5,46 & 97,61 & 4,72 & 4,02 \\
\hline Total & 189 & 862 & 5,06 & 94,27 & 5,24 & 4,18 \\
\hline
\end{tabular}

